\title{
Transversals in Superdisjoint $T(3)$-families of Translates
}

\author{
Aladár Heppes
}

Received: 12 November 2009 / Revised: 14 May 2010 / Accepted: 25 August 2010 /

Published online: 14 September 2010

(C) Springer Science+Business Media, LLC 2010

\begin{abstract}
Let $K$ denote an oval, a centrally symmetric compact convex domain with non-empty interior. A family of translates of $K$ is said to have property $T(k)$ if for every subset of at most $k$ translates there exists a common line transversal intersecting all of them. Property $T$ means that a transversal exists for all members of the family. Two translates, $K_{i}$ and $K_{j}$ are said to be $\varphi$-disjoint, $\varphi>0$, if the concentric $\varphi$-enlarged copies of $K_{i}$ and $K_{j}$ are disjoint. It is well known that in a $\varphi$-disjoint family of congruent $\operatorname{discs} T(3) \Rightarrow T$ if $\varphi>\sqrt{2}$, and $T(3) \nRightarrow T$ if $\varphi<\sqrt{2}$. In this note finite $\varphi$-disjoint $T$ (3)-families of translates of an oval will be investigated.
\end{abstract}

Keywords Transversal Helly $\cdot$ Superdisjointness · Critical factor · Total separability

\section{Introduction}

Helly type problems for line transversals of families of convex domains have been studied by a number of authors. The interested reader is referred to the survey papers [2, 3, 9] or [5]. In the present note finite families of translated copies of a compact convex domain are considered in the Euclidean plane.

Definitions and notations Throughout the paper the term oval will be used for a centrally symmetric compact convex domain in the plane with non-empty interior. (The assumption of central symmetry is no restriction when transversal problems are considered in the plane. For the details of the reduction see e.g. [8]. Clearly, neither does affinity influence the validity of the related statements.) Let $K$ be an oval centered at the origin $O$ and $\mathcal{F}=\left\{K_{i}=K+c_{i}, i=1, \ldots, n\right\}$ a family of a finite

A. Heppes ( $\bowtie)$

Rényi Institute of the Hungarian Academy of Sciences, Reáltanoda u. 13-15, 1053 Budapest, Hungary

e-mail: aheppes@renyi.hu 
number of translated copies, translates, of $K . \mathcal{C}=\left\{c_{i}, i=1, \ldots, n\right\}$ denotes the set of the centers. The term $K$-distance and notation $|p-q|_{K}$ will be used for the distance between points $p$ and $q$ measured in the norm induced by $K$. The width $w(X)$, ( $K$-width $w_{K}(X)$ ) of a closed set is the minimum of the distances ( $K$-distances) of pairs of parallel support lines of $X$. The diameter ( $K$-diameter) of a closed set is the maximum of the distances ( $K$-distances) between pairs of points in the set.

We consider families of translates of an oval $K$. Such a family $\mathcal{F}$ is a $T$-family (or it has property $T$ ) if there exists a straight line, a transversal, intersecting all members of $\mathcal{F} . \mathcal{F}$ is a $T(k)$-family (it has property $T(k)$ ) for some integer $k \geq 3$, if every subfamily of at most $k$ members of $\mathcal{F}$ has property $T$. We also say that the family has stabbing level $k . \mathcal{F}$ is a $T$-family if and only if the set of the centers can be covered with a strip of $K$-width 2 .

We say that $\mathcal{F}$ is totally separable if there exists a direction such that any line parallel with this direction intersects at most one member of $\mathcal{F}$.

For a real number $\varphi>0$ let $\varphi K$ denote the oval obtained by scaling $K$ by factor $\varphi$. Two ovals $K_{i}=K+c_{i}$ and $K_{j}=K+c_{j}$ are $\varphi$-disjoint if $\varphi K+c_{i}$ and $\varphi K+c_{j}$ are disjoint. If $\varphi>1$ we speak about superdisjoint ovals.

The family $\mathcal{F}$ is $\varphi$-disjoint if its members are mutually $\varphi$-disjoint. In a $\varphi$-disjoint family of translates of an oval $K$ the $K$-distance between any two centers is more than $2 \varphi$. We say that $\varphi_{k}(K)$ is the critical factor to the oval $K$ at stabbing level $k$ if in a $\varphi$-disjoint family of translates of $K T(k) \Rightarrow T$ if $\varphi>\varphi_{k}(K)$ and $T(k) \nRightarrow T$ if $\varphi<\varphi_{k}(K)$.

\section{Results}

From the many old results connected with transversal Helly numbers we cite only those which are most related to the topic discussed here. (See [4] and [1].)

Theorem 2.1 Let $K_{\circ}$ be a disc. Then $\varphi_{3}\left(K_{\circ}\right)=\sqrt{2}$.

In other words if $\mathcal{F}$ is a finite family of $\varphi$-disjoint congruent discs with $\varphi \geq \sqrt{2}$ then $T(3) \Rightarrow T$ and $\varphi=\sqrt{2}$ is the smallest value with this property. Our goal is to extend the investigation to cases when ovals, different from discs, are also considered. We prove the following theorems:

Theorem 2.2 Let $K$ be an oval. Then $\varphi_{3}(K) \leq 2$. Equality holds only when $K$ is a parallelogram.

It can also be asked which is the smallest $\varphi$ implying a transversal for some oval. The answer is given in

Theorem 2.3 Let $K$ be an oval. Then $\varphi_{3}(K) \geq \sqrt{2}$. Equality holds, e.g., when $K$ is a disc.

These theorems, generalizing Theorem 2.1, show that the circle is (one of) the "most convenient" and the square is the "least convenient" oval. 
The next theorem claims that the possible $\varphi_{3}(K)$ values make up an interval.

Theorem 2.4 To any $\varphi^{*} \in[\sqrt{2}, 2]$, exists an oval $K^{*}$ such that $\varphi_{3}\left(K^{*}\right)=\varphi^{*}$.

\section{Proofs}

The following result of Holmsen (see Proposition 3 in [6]) will be referred to in the proofs.

Lemma 3.0 If in a family of disjoint translates of an oval every triple of translates is totally separable then $T(3) \Rightarrow T$.

\subsection{Proof of Theorem 2.2}

In view of Lemma 3.0, to show that $\varphi=2$ implies $T(3) \Rightarrow T$ for any oval, it will be sufficient to prove that whenever $\varphi=2$ holds then every triple is totally separable.

A chord of direction $d$ of an oval will be called, by definition, a mid-chord of direction $d$ if it is lying half way between a $d$-directional support line and the center of the oval. The convex hull of the two mid-chords of direction $d$ is a parallelogram whose two sides are parallel with $d$. The direction of the other two sides will be called the direction conjugate to $d$.

Next we prove

Lemma 3.1 Let $K_{1}$ and $K_{2}$ be two 2-disjoint translates of the oval $K$. If a straight line of direction $d$ is intersecting both translates then $K_{1}$ and $K_{2}$ can be separated by a line of direction conjugate to $d$.

Proof of Lemma 3.1 Since the claim of the lemma is not affected by an affine transformation we can suppose without loss of the generality that the common transversal of the two ovals is horizontal and the direction conjugate to the horizontal direction is vertical. We also assume that the vertical width of $K$ is 2 , and the length of a horizontal mid-chord is $1, K_{1}$ is centered at the origin and $K_{2}$ is centered at point $c_{2}\left(x_{2}, y_{2}\right)$.

Case symmetry allows us to assume that $x_{2} \geq 0$ and $y_{2} \geq 0$. Let $A_{1}$ denote a boundary point of $K_{1}$ on the upper horizontal support line $y=1$, further let $A_{2}$ and $A_{3}$ be the endpoints of the associated mid-chord. Points $A_{i}^{\prime}$ denote the images of $A_{i}$, $i=1,2,3$ obtained by reflection in $O$. The convex hull of $A_{2}, A_{3}, A_{2}^{\prime}$ and $A_{3}^{\prime}$ is an axis parallel square of unit side length thus we have $A_{1}\left(a_{1}, 1\right), A_{2}(-1 / 2,1 / 2)$, $A_{3}(1 / 2,1 / 2)$ (see Fig. 1). In this special case our task is to show that $K_{1}$ and $K_{2}$ can be separated by a vertical line.

On one hand $y_{2} \leq 2$ must hold for the center $c_{2}$ of $K_{2}$ since $K_{1}$ and $K_{2}$ have a common horizontal transversal. On the other hand the 2-disjointness property implies $x_{2}>2$ since no center may belong to the enlarged oval $4 K^{\prime}$, where $K^{\prime}$ denotes the convex hull of the six boundary points $A_{1}, A_{2}, A_{3}, A_{1}^{\prime}, A_{2}^{\prime}$ and $A_{3}^{\prime}$.

We claim that $K_{1}$ and $K_{2}$ are separated by the vertical line $x=x_{2} / 2$. Suppose that it is not so. Then both (centrally symmetric) ovals $K_{1}$ and $K_{2}$ intersect this vertical 
Fig. 1 Ovals $K^{\prime}$ and $4 K^{\prime}$

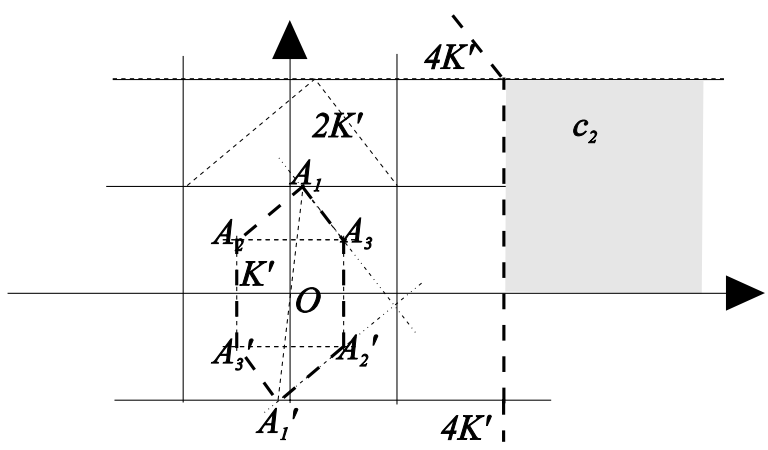

Fig. 2 A $T$ (3)-family of $(2-\varepsilon)$-disjoint squares with no transversal

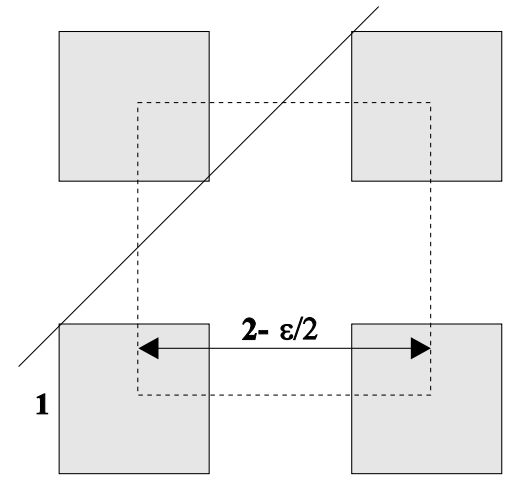

line, moreover $K_{1}$ has a point $A_{4}\left(x_{2} / 2, a_{4}\right)$ on the vertical line $x=x_{2} / 2$. Because of the convexity of $K$ this point cannot be above the line $A_{1} A_{3}$, and it cannot be below the line $A_{1}^{\prime} A_{2}^{\prime}$ either. Easily, these conditions imply that either the set of inequalities $0 \leq a_{1}<-a_{4}<a_{1}$ or the set $0 \leq-a_{1}<a_{4}<-a_{1}$ must be valid. Clearly, neither set can be fulfilled.

Consequently, a vertical line $x=x_{2} / 2(>1)$ is separating $K_{1}$ and $K_{2}$. By this Lemma 3.1 is proved.

Lemma 3.1 implies that whenever a subset of our 2-disjoint family $\mathcal{F}$ has a transversal (of direction $d$ ) this subset is totally separable (in direction $d^{\prime}$ conjugate to $d$ ). Since in our case a transversal line exists to every triple of ovals the conditions to apply Lemma 3.0 are fulfilled. This proves the inequality $\varphi_{3}(K) \leq 2$. Easily, even $\varphi_{3}(K)<2$ holds if $K$ is not a parallelogram.

To prove that the inequality in the theorem is sharp we show that $\varphi \geq 2$ is necessary when $K$ is a square.

Let $0<\varepsilon<1$, and $\varphi=2-\varepsilon>1$. The family consisting of the four axis parallel squares of unit side length centered at the vertices of an axis parallel square of side length $2-\varepsilon / 2$ provides an example for a $(2-\varepsilon)$-disjoint family which has property $T$ (3) without having a transversal (Fig. 2). This concludes the proof of Theorem 2.2. 
Fig. 3 Non-separable triple of $\varphi$-disjoint ovals with $\varphi>2$

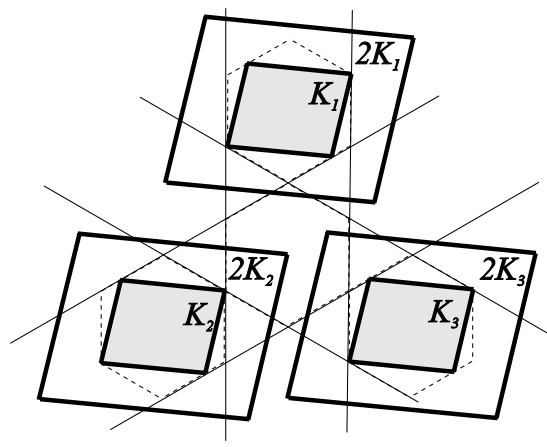

Remarks (1) The assumption of 2-disjointness in itself is not sufficient to guarantee the separation of the ovals. (The three rhombs displayed on Fig. 3 show that even $\varphi>2$ does not necessarily imply separation.) It is the simultaneous requirement of 2-disjointness and property $T(3)$ which yields it.

(2) J. Jerónimo noticed (private communication) that a different proof of the sharp upper bound could be based on Theorem 2.1 and John's theorem [7] claiming that every centrally symmetric domain $K$ can be "sandwiched" between two ellipses $E$ and $\lambda E$, where $\lambda \leq \sqrt{2}$ and $\lambda<\sqrt{2}$ except if $K$ is a parallelogram.

\subsection{Proof of Theorem 2.3}

To prove the theorem it will be shown that for any oval $K$ and any $\varepsilon>0$ there exists a $T$ (3)-family of four translates which are $\varphi$-disjoint with $\varphi=\sqrt{2}-\varepsilon$ such that there is no straight line intersecting all four of them.

Let $K$ be symmetric to the origin $O$ and $R(d)$ a rectangle circumscribed about $K$ such that the directions of the sides of $R(d)$ are $d$ and $d^{\prime}$, - orthogonal to $d-$, respectively. We denote by $\mathcal{A}_{d}$ the affine transformation which maps $R(d)$ into a unit square $S(d)$ keeping the center and the directions of the sides unchanged. (The square $S(d)=\mathcal{A}_{d}[R(d)]$ is circumscribed about the mapped oval $K(d)=\mathcal{A}_{d}[K]$.)

Let $S^{\prime}(d)$ denote the square obtained from $S(d)$ by a $\pi / 4$ rotation about its center $O$. Let $u(d)$ and $v(d)$ denote the signed distances of two adjacent sides of $S^{\prime}(d)$ from the nearest parallel support line of $K(d)$. Clearly, $u(d)$ and $v(d)$ are continuous functions of $d$ and, while the direction $d$ is changed from the initial direction to its orthogonal $d^{\prime}$, these two distance values exchange roles. Consequently there exists a direction $d_{0}$ for which $u\left(d_{0}\right)=v\left(d_{0}\right)$. For this direction $d_{0}$ there exists a factor $\alpha$ such that $\alpha S^{\prime}\left(d_{0}\right)$ is a square circumscribed about $K(d)$ as well as $S\left(d_{0}\right)$ is (Fig. 4).

Since there are boundary points of $K\left(d_{0}\right)$ on every side of both squares it holds $\sqrt{2} / 2 \leq \alpha \leq \sqrt{2}$. We can assume that $\alpha \geq 1$ else a stretching by $1 / \alpha$ and exchanging the roles of $d$ and $d^{\prime}$ can be applied.

Without loss of the generality we may assume that $d_{0}$ is horizontal. Consider the family consisting of the four translates $K\left(d_{0}\right)+( \pm \alpha / \sqrt{2}, \pm \alpha / \sqrt{2})$ (Fig. 5).

In this family there is a single transversal to every triple of ovals but none to the whole family. As $\alpha \in[1, \sqrt{2}]$ and $\varphi \geq \sqrt{2} \alpha$ the distance between any pair of centers is at least $\sqrt{2}$ and the family is $\varphi$-disjoint for any $\varphi<\sqrt{2}$. 
Fig. 4 Two squares "tightly" circumscribed about the oval

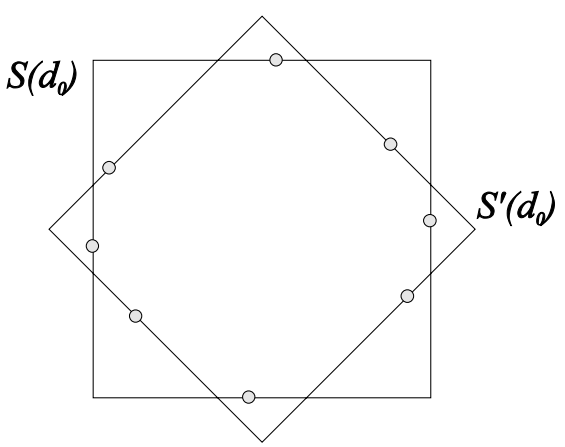

Fig. $5 T$ (3) quadruple of the circumscribed octagons

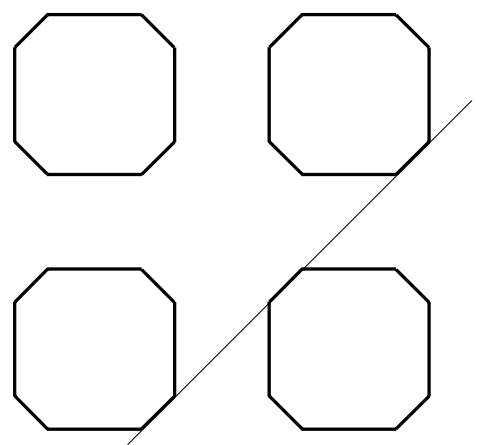

This shows that $\varphi \geq \sqrt{2}$ is necessary to imply $T(3) \Rightarrow T$ whatever oval $K$ we start with.

Remark By Theorems 2.2 and 2.3 we have proved for the critical factor $\varphi_{3}(K)$ the inequalities

$$
\sqrt{2} \leq \varphi_{3}(K) \leq 2 .
$$

Both bounds on $\varphi_{3}$ are sharp. The first one for the ellipses and the second one for the parallelograms.

\subsection{Proof of Theorem 2.4}

Let $\varphi^{*} \in[\sqrt{2}, 2]$ and $S(s)$ an axis parallel $O$-centered square of side length $s$. For $s=\sqrt{8} / \varphi^{*}$ let $K(s)$ denote the intersection of $S(s)$ and the $O$-centered unit circle. To prove the theorem it will be shown that the critical factor of $K(s)$ is $\varphi^{*}$.

Consider the family of the four translates $K_{i}(s), i=1, \ldots, 4$, of $K(s)$ centered at the vertices of $S(\sqrt{8})$. Clearly, this family is $\varphi$-disjoint for any $\varphi<\varphi^{*}$ since the $\varphi^{*}$-enlarged ovals are tangent (Fig. 6).

As this is a $T(3)$-family without transversal we have

$$
\varphi_{3}(K(s)) \leq \varphi^{*} .
$$


Fig. $6 T(3)$ family of $K(s)$ ovals having no transversal

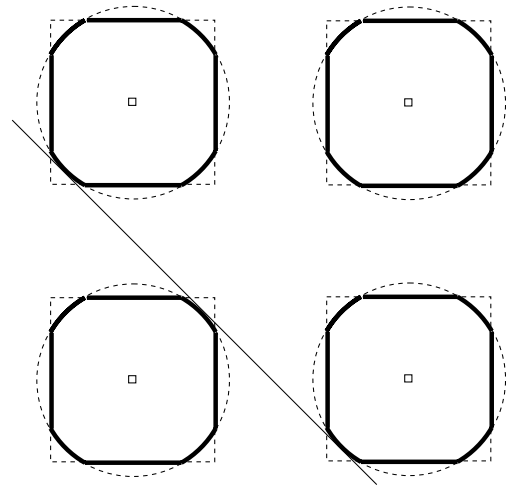

On the other hand if the family of $K(s)$ ovals is $\varphi$-disjoint for any $\varphi \geq \varphi^{*}$ then the mutual distance between the centers is greater than $\sqrt{8}$. Consequently, even the circumcircles of an arbitrary triple of the ovals is totally separable in the direction orthogonal to the longest side of the triangle of centers. Then combining this with the $T$ (3) property of the family Lemma 3.0 can again be applied and the missing relation

$$
\varphi_{3}(K(s)) \geq \varphi^{*}
$$

follows.

Remark The boundary of the oval used above consists of four segments and four circle arcs. The above proof works without change if $K(s)$ can be replaced by the convex hull of the midpoints of the segments and arcs.

For the limiting case of $\varphi^{*}=\sqrt{2}$ this leads to a regular octagon showing that the disc is not the unique oval of critical factor $\sqrt{2}$.

Acknowledgements This research was partially supported by Hungarian Science Foundation OTKA Grant No. K68398. The author wishes to express his thanks to the unknown referee for his/her comments, especially for raising the author's attention to the fact that Theorem 2.2 was previously proved by Grünbaum (see [4], Corollary 4).

\section{References}

1. Bezdek, K., Bisztriczky, T., Csikós, B., Heppes, A.: On the transversal Helly numbers of disjoint and overlapping discs. Arch. Math. 87, 86-96 (2006)

2. Eckhoff, J.: Helly, Radon and Carathéodory type theorems. In: Gruber, P.M. (ed.) Handbook of Convex Geometry, vol. A, pp. 389-448. North-Holland, Amsterdam (1993)

3. Goodman, J.E., Pollack, R., Wenger, R.: Geometric transversal theory. In: Pach, J. (ed.) New Trends in Discrete and Computational Geometry, pp. 163-198. Springer, Berlin (1993)

4. Grünbaum, B.: Common transversals for families of sets. J. Lond. Math. Soc. 35, 408-416 (1960)

5. Holmsen, A.: Recent progress on line transversals to families of translated ovals. In: Goodman, J.E., Pach, J., Pollack, R. (eds.) Contemporary Mathematics, vol. 453. AMS, Providence (2008)

6. Holmsen, A.: New bounds on the Katchalski-Lewis transversal problem. Discrete Comput. Geom. 29, 395-408 (2003) 
7. John, F.: Extremum problems with inequalities as subsidiary conditions. In: Studies and Essays Presented to R. Courant on his 60th Birthday, 8 January 1948, pp. 187-204. Interscience, New York (1948)

8. Tverberg, H.: Proof of Grünbaum's conjecture on common transversals for translates. Discrete Comput. Geom. 4, 191-203 (1989)

9. Wenger, R.: Helly-type theorems and geometric transversals. In: Goodman, J.E., O'Rourke, J. (eds.) Handbook of Discrete and Computational Geometry, 2nd edn. CRC Press, Boca Raton (2004), Chap. 4 